\begin{tabular}{|c|l|}
\hline Title & Localized vibrational modes in superlattices \\
\hline Author(s) & Tamura, Shin-ichiro \\
\hline Citation & $\begin{array}{l}\text { Physical Review B, 39(2), 1261-1269 } \\
\text { https://doi.org/10.1103/PhysRevB.39.1261 }\end{array}$ \\
\hline Issue Date & 1989-01-15 \\
\hline Doc URL & http://hdl.handle.net/2115/5949 \\
\hline Rights & Copyright $\odot$ 1989 A merican Physical Society \\
\hline Type & article \\
\hline File Information & PRB39-2pdf.pdf \\
\hline
\end{tabular}

Instructions for use 


\title{
Localized vibrational modes in superlattices
}

\author{
Shin-ichiro Tamura \\ Department of Engineering Science, Hokkaido University, Sapporo 060, Hokkaido, Japan
}

(Received 12 September 1988)

\begin{abstract}
The effect of an impurity cell on the vibrational properties of superlattices is studied theoretically. The continuous equation governing the elastic-wave motion in a superlattice is transformed exactly to a discrete form which is analogous to the equation for the displacement in one-dimensional discrete lattices. With the use of this equation isolated frequencies associated with the vibrations localized near the impurity cell are predicted in the band gaps at the center and boundary of the folded Brillouin zone of the host superlattice. Numerical calculation reveals further the existence of impurity states in the intrazone gaps due to intermode Bragg reflection. The calculated phonon transmission rate shows local enhancements due to these localized states, suggesting their observability by phonon spectroscopic experiment with a quasimonochromatic phonon detector.
\end{abstract}

\section{INTRODUCTION}

Recently, there has been an accumulation of studies on the vibrational properties of superlattices (SL's). ${ }^{1-15}$ However, they are mainly restricted to those in perfect, periodic, ${ }^{1-12}$ and quasiperiodic systems. ${ }^{13-16}$ The vibrations in disordered SL's should also provide an interesting subject of both experimental and theoretical researches. The lattice vibrations in disordered crystals analogous to the corresponding problem for the SL's have been studied for more than 20 years. ${ }^{17}$ It is well established that even a small concentration of defects in a crystal lattice can radically alter the frequencies of the normal modes of vibration as well as the pattern of atomic displacement associated with these modes. The same kinds of effects are also expected to occur when the layers with different thickness or different constituents, i.e., impurity cells, are embedded in an otherwise perfect SL.

More specifically, when a sufficiently light-mass defect is added substitutionally to a crystal, it causes a mode outside the band of allowed frequencies of the perfect crystal. This is a local mode of the vibration whose amplitude is strongly localized in the vicinity of the defect. In a SL with an impurity cell the similar local mode, if any are present, should appear in frequency gaps produced at the center, boundary, and even inside the folded Brillouin zone of the SL.

The purpose of the present paper is to analyze the existence and nature of the localized vibrational modes in the "impure" SL with an isolated impurity cell. This study will also provide a clue to investigating the vibrational properties of intentionally disordered, random SL's because the effects of a finite concentration of impurity cells can be deduced rather well from a knowledge of the effects of only one or two isolated defects.

Through this work we employ the continuum model for lattice vibrations, ${ }^{18}$ which has successfully explained a number of recent experiments on acoustic phonons in SL's. 3,7,9,10 The basic equations of this model can be transformed into a discrete form which is more convenient for analyzing vibrations in an impure SL. Then, the equation for determining the frequencies of normal modes which are perturbed by the introduction of the impurity cell is derived. Generally, this equation can only be solved numerically. However, in the particular cases of practical interest it can be studied analytically and the existence of localized modes of vibration is predicted in the frequency gaps of the host SL.

One of the physical quantities readily accessible by phonon spectroscopic experiments related to this subject is a phonon transmission rate. The calculated transmission rate in the impure SL's with a finite number of cells reveals sharp enhancement in the frequency regions corresponding to forbidden gaps of the host SL, i.e., the regions with vanishing phonon transmission. The angular dependence of the transmission shows the similar local enhancement in the gaps due to intermode Bragg reflection, ${ }^{8,9}$ indicating the presence of the localized mode also within the frequency gaps inside the folded Brillouin zone of pure SL's. Thus the observability of the localized vibrational states by a phonon transmission experiment will be possible.

\section{FORMULATION}

The SL system we shall consider is shown schematically in Fig. 1. An impurity cell denoted by $X$ consisting of generally different $x$ and $y$ layers with thickness $d_{x}$ and $d_{y}\left(d_{x}+d_{y} \equiv D^{\prime}\right)$ is embedded in the periodic array of alternating $a$ and $b$ layers with thickness $d_{a}$ and $d_{b}$, respectively. This means that the host SL is constructed by unit cells denoted by $A$ with the periodicity $D=d_{a}+d_{b}$. We can treat the case of a single impurity layer by assuming that $x$ and $y$ are the same constituents, or by putting $d_{x}=0$ or $d_{y}=0$.

In our formulation the continuum model for the lattice vibration is assumed. ${ }^{18}$ For simplicity we shall consider the case where the wave vector of the vibration (phonon) is parallel to the growth direction of the SL. (The more general case will be discussed in Sec. III.) In this case the lattice vibration is conveniently described by a twocomponent column vector $\mathbf{W}(z)=(U(z), S(z))^{t}$, where $U$ 


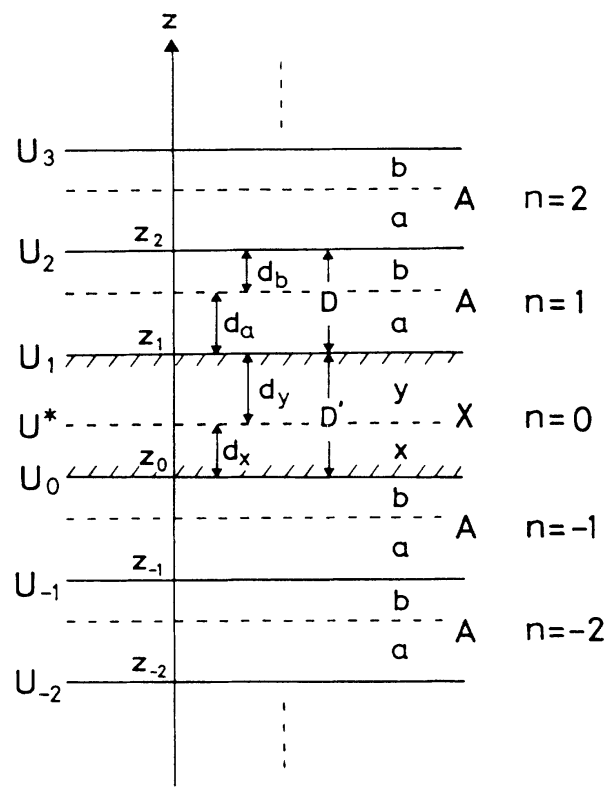

FIG. 1. Schematic superlattice (SL) system. An impurity cell $X$ consisting of binary $x$ and $y$ layers occupies the $n=0$ site of the otherwise perfect, periodic superlattice consisting of alternating $a$ and $b$ layers. The width $D=d_{a}+d_{b}$ of the unit cell $A$ gives the periodicity of the host SL. The vibrational amplitude at the cell interface $z=z_{n}$ is denoted by $U_{n}$. $U^{*}$ indicates the vibrational amplitude at the interface between $x$ and $y$ layers.

and $S$ are the nonvanishing components of the displacement and stress fields, respectively, and $t$ represents a transposition. ${ }^{9,18}$ Explicitly, $U$ and $S$ are written as

$$
\begin{aligned}
& U(z)=c_{I}^{(+)} e^{i k_{I} z}+c_{I}^{(-)} e^{-i k_{I} z}, \\
& S(z)=i \omega Z_{I}\left(c_{I}^{(+)} e^{i k_{I} z}-c_{I}^{(-)} e^{i k_{I} z}\right),
\end{aligned}
$$

where $I$ is the index specifying different layers (i.e., $I=a$, $b, x$, and $y), c^{(+)}\left(c^{(-)}\right)$is the amplitude of the $+\mathbf{z}(-\mathbf{z})$-propagating wave, $k$ is the wave number, $Z=\rho v$ is the acoustic impedance with $\rho$ the mass density and $v$ the sound velocity, and $\omega=k v$ is the angular frequency.

At each interface of adjacent layers $W$ should be continuous. With the use of this condition we find that $\mathbf{W}$ changes to $\underline{T}_{A} \mathrm{~W}\left(\underline{T}_{X} \mathrm{~W}\right)$ after the propagation of a phonon through an $A($ an $X)$ cell. The "transfer matrix" $\underline{T}_{A}$ is unimodular, and defined by ${ }^{15}$

$$
\underline{T}_{A}=\underline{t}_{b} \underline{t}_{a} \equiv\left(\begin{array}{ll}
\lambda_{A} & \sigma_{A} \\
\zeta_{A} & \mu_{A}
\end{array}\right),
$$

with

$$
\underline{t}_{a}=\left(\begin{array}{cc}
\cos \alpha & \frac{1}{i \omega Z_{a}} \sin \alpha \\
-i \omega Z_{a} \sin \alpha & \cos \alpha
\end{array}\right],
$$

where $\alpha=k_{a} d_{a}$, and $\underline{t}_{b}$ is defined similarly with $\alpha$ and $Z_{a}$ replaced by $\beta=k_{b} d_{b}$ and $Z_{b}$, respectively. Explicit expressions for the elements of $\underline{T}_{A}$ are given by

$$
\begin{aligned}
\lambda_{A} & =\cos \alpha \cos \beta-\left(Z_{a} / Z_{b}\right) \sin \alpha \sin \beta, \\
\sigma_{A} & =\frac{1}{i \omega Z_{a}} \sin \alpha \cos \beta+\frac{1}{i \omega Z_{b}} \cos \alpha \sin \beta, \\
\zeta_{A} & =-i \omega Z_{a} \sin \alpha \cos \beta-i \omega Z_{b} \cos \alpha \sin \beta, \\
\mu_{A} & =\cos \alpha \cos \beta-\left(Z_{b} / Z_{a}\right) \sin \alpha \sin \beta,
\end{aligned}
$$

and they satisfy $\operatorname{det} \underline{T}_{A}=\lambda_{A} \mu_{A}-\sigma_{A} \zeta_{A}=1$. The transfer matrix $\underline{T}_{X}$ related to the impurity cell is also defined similarly as

$$
\underline{T}_{X}=\left(\begin{array}{ll}
\lambda_{X} & \sigma_{X} \\
\zeta_{X} & \mu_{X}
\end{array}\right),
$$

where the matrix elements are written in the form of Eq. (4) in terms of parameters relevant to the impurity layers.

Now, introducing $\mathbf{W}_{n}=\left(U_{n}, S_{n}\right)^{t} \equiv \mathbf{W}\left(z_{n}\right)$, we can write

$$
\mathbf{W}_{n+1}=\underline{T}_{n} \mathbf{W}_{n},
$$

where

$$
\underline{T}_{n}=\left(\begin{array}{ll}
\lambda_{n} & \sigma_{n} \\
\zeta_{n} & \mu_{n}
\end{array}\right)
$$

is the generalization of Eqs. (2) and (5), that is, the transfer matrix $\underline{T}_{n}$ is related to the $n$th unit cell of the SL and $\underline{T}_{n}=\underline{T}_{A}$ for $n \neq 0$ and $\underline{T}_{n}=\underline{T}_{X}$ for $n=0$ (see Fig. 1). Our goal is to derive the equation involving only $U_{n}$ 's, i.e., the equation for displacement amplitudes at cell interfaces.

At the interface $z=z_{n}$, Eq. (1) takes the form

$$
\mathbf{W}_{n}=\underline{P}_{n} \text {, }
$$

where $\mathbf{C}_{n}=\left(c_{I}^{(+)} e^{i k_{I} z_{n}}, c_{I}^{(-)} e^{-i k_{I} z_{n}}\right)^{t} \equiv\left(c_{n}^{(+)}, c_{n}^{(-)}\right)^{t}$, and

$$
\underline{P}=\left[\begin{array}{cc}
1 & 1 \\
i \omega Z_{I} & -i \omega Z_{I}
\end{array}\right) \text {. }
$$

In order to fix the index $I$ in the above equations an infinitesimal positive number may be added to or subtracted from $z=z_{n}$. Of course the result does not depend on this choice because of the continuity of $\mathbf{W}$ at each interface. Equation (8) allows us to rewrite Eq. (6) as

$$
\mathbf{W}_{n}=\underline{R}_{n} \mathbf{C}_{n+1},
$$

where

$$
\underline{R}_{n}=\underline{T}_{n}^{-1} \underline{P}
$$

is obtained by combining Eqs. (6) and (8). Hence, from Eqs. (8) and (10), we have

$$
U_{n}=c_{n}^{(+)}+c_{n}^{(-)}=\left(\underline{R}_{n}\right)_{11} c_{n+1}^{(+)}+\left(\underline{R}_{n}\right)_{12} c_{n+1}^{(-)}
$$

and

$$
U_{n-1}=\left(\underline{R}_{n-1}\right)_{11} c_{n}^{(+)}+\left(\underline{R}_{n-1}\right)_{12} c_{n}^{(-)} .
$$


These equations are summarized as

$$
\mathbf{V}_{n}=\underline{Y}_{n-1} \mathbf{C}_{n},
$$

where $\mathbf{V}_{n}=\left(U_{n}, U_{n-1}\right)^{t}$ and

$$
\underline{Y}_{n}=\left[\begin{array}{cc}
1 & 1 \\
\left(\underline{R}_{n}\right)_{11} & \left(\underline{R}_{n}\right)_{12}
\end{array}\right] \text {. }
$$

Eliminating $\mathbf{C}_{n}$ from (14) with the aid of Eqs. (8) and (10) we have the equation for two consecutive V's,

$$
\underline{T}_{n}^{-1} \underline{P} \underline{Y}_{n}^{-1} \mathbf{V}_{n+1}=\underline{P} \underline{Y}_{n-1}^{-1} \mathbf{V}_{n}
$$

By noting that

$$
\underline{P} \underline{Y}_{n}^{-1}=\left[\begin{array}{cc}
1 & 0 \\
\mu_{n} / \sigma_{n} & -1 / \sigma_{n}
\end{array}\right],
$$

Eq. (16) is expressed explicitly as

$$
\begin{aligned}
\left(\begin{array}{cc}
0 & 1 \\
1 / \sigma_{n} & -\lambda_{n} / \sigma_{n}
\end{array}\right)\left(\begin{array}{c}
U_{n+1} \\
U_{n}
\end{array}\right) & \\
= & \left(\begin{array}{cc}
1 & 0 \\
\mu_{n-1} / \sigma_{n-1} & -1 / \sigma_{n-1}
\end{array}\right)\left(\begin{array}{c}
U_{n} \\
U_{n-1}
\end{array}\right) .
\end{aligned}
$$

Thus we have the discrete equation governing the displacement amplitude at interfaces of adjacent unit cells,

$$
\frac{1}{\sigma_{n}} U_{n+1}+\frac{1}{\sigma_{n-1}} U_{n-1}=\left(\frac{\lambda_{n}}{\sigma_{n}}+\frac{\mu_{n-1}}{\sigma_{n-1}}\right) U_{n} .
$$

The above procedure for obtaining Eq. (19) is similar to the one for transforming the one-dimensional Schrödinger equation with multiple scattering potentials to a discrete, tight-binding form. ${ }^{19}$

In the perfect, periodic SL consisting of only $A$-type cells, Eq. (19) is reduced to

$$
U_{n+1}+U_{n-1}=\left(\lambda_{A}+\mu_{A}\right) U_{n} .
$$

Substituting $U_{n}=e^{i n Q}$ into this equation, we obtain

$$
2 \cos Q=\lambda_{A}+\mu_{A} \equiv \Omega_{A}(\omega) .
$$

By putting $Q=q D$, this gives the well-known phonon dispersion relation $\omega=\omega(q)$ in the periodic SL. ${ }^{18}$ With these results the study of the effect of an impurity cell embedded in a periodic SL becomes analogous to the corresponding problem for one-dimensional lattices.

For the following discussion it is convenient to write Eq. (19) in the form

$$
\begin{aligned}
\left(1+\delta K_{n}\right) U_{n+1}+\left(1+\delta K_{n-1}\right) U_{n-1} & \\
& =\left(\Omega_{A}+\delta J_{n}+\delta M_{n-1}\right) U_{n},
\end{aligned}
$$

where $\quad \delta K_{n}=\left(\sigma_{A} / \sigma_{n}\right)-1, \quad \delta J_{n}=\left[\left(\lambda_{n} / \sigma_{n}\right)-\left(\lambda_{A} /\right.\right.$ $\left.\left.\sigma_{A}\right)\right] \sigma_{A}$, and $\delta M_{n}=\left[\left(\mu_{n} / \sigma_{n}\right)-\left(\mu_{A} / \sigma_{A}\right)\right] \sigma_{A}$. Note that $\delta K_{n}, \delta J_{n}$, and $\delta M_{n}$ are nonzero only at the impurity-cell site, or for $n=0$. Thus it holds that

$$
\begin{aligned}
& \delta K_{n}=\delta K \delta_{n, 0}=\left(\frac{\sigma_{A}}{\sigma_{X}}-1\right) \delta_{n, 0}, \\
& \delta J_{n}=\delta J \delta_{n, 0}=\sigma_{A}\left(\frac{\lambda_{X}}{\sigma_{X}}-\frac{\lambda_{A}}{\sigma_{A}}\right) \delta_{n, 0}, \\
& \delta M_{n}=\delta M \delta_{n, 0}=\sigma_{A}\left(\frac{\mu_{X}}{\sigma_{X}}-\frac{\mu_{A}}{\sigma_{A}}\right) \delta_{n, 0} .
\end{aligned}
$$

In order to solve Eq. (22) we formally express it as ${ }^{17}$

$$
\sum_{m}\left(L_{n m}+\delta L_{n m}\right) U_{m}=0
$$

where

$$
\begin{aligned}
L_{n m}= & \delta_{n, m-1}+\delta_{n, m+1}-\Omega_{A}(\omega) \delta_{n, m}, \\
\delta L_{n m}= & \delta K\left(\delta_{n, 0} \delta_{n, m-1}+\delta_{n, 1} \delta_{n, m+1}\right) \\
& -\left(\delta J \delta_{n, 0}+\delta M \delta_{n, 1}\right) \delta_{n, m} .
\end{aligned}
$$

In terms of $L_{n m}$, Eq. (20) for the perfect, periodic SL takes the form

$$
\sum_{m} L_{n m} U_{m}=0 \text {. }
$$

Now, we introduce the Green's function $G_{n m}$ defined by

$$
\sum_{l} L_{n l} G_{l m}=\delta_{n, m}
$$

or equivalently,

$$
G_{n+1 m}+G_{n-1 m}-\Omega_{A}(\omega) G_{n m}=\delta_{n, m} .
$$

Expanding $G_{n m}$ in a Fourier series, we have for the ideal SL with an infinite repetition of $A$-type cells,

$$
\begin{aligned}
G_{n m}\left(\Omega_{A}\right) & =\frac{1}{\pi} \int_{0}^{\pi} \frac{\cos [(n-m) Q]}{2 \cos Q-\Omega_{A}(\omega)} d Q \\
& \equiv g_{n-m}\left(\Omega_{A}\right) .
\end{aligned}
$$

The integration can be performed analytically and we find $^{20}$

$g_{n}\left(\Omega_{A}\right)=-\frac{1}{\left(\Omega_{A}^{2}-4\right)^{1 / 2}}\left[\frac{\Omega_{A}-\left(\Omega_{A}^{2}-4\right)^{1 / 2}}{2}\right]^{|n|}$,

where this result is obtained for real $\Omega_{A}$ larger than 2 . Now, $g_{n}$ has a branch cut along a straight line $-2<\Omega_{A}<2$ and, by analytic continuation, the resulting expression for $g_{n}$ should be valid throughout the physical sheet of the complex $\Omega_{A}$ plane. Here, we note that for $\Omega_{A}$ which is real and satisfies $\left|\Omega_{A}\right|>2$, the modulus of

$$
\epsilon=\left[\Omega_{A}-\left(\Omega_{A}^{2}-4\right)^{1 / 2}\right] / 2
$$

is smaller than unity, and hence $g_{n}$ decreases exponentially, i.e., $g_{n} \sim \epsilon^{|n|}$, with increasing $|n|$.

Next, we consider the SL with $N-1 A$-type cells on either side of the impurity cell and express the $2 N \times 2 N$ matrices $\left(G_{n m}\right)$ and $\left(\delta L_{n m}\right)$ by $\underline{G}$ and $\delta \underline{L}$, and the $2 N$ component column vector 


$$
\left(U_{-N+1}, U_{-N+2}, \ldots, U_{N}\right)^{t}
$$

by $\mathbf{U}$. Thus, from Eq. (24), we have

$$
(\underline{1}+\underline{G} \delta \underline{L}) \mathbf{U}=\mathbf{0},
$$

where 1 is the $2 N \times 2 N$ matrix. Because $\delta \underline{L}$ has only four nonvanishing components, we introduce the $2 \times 2$ matrix $\delta \underline{l}$ defined by

$$
\delta \underline{l}=\left(\begin{array}{cc}
-\delta J & \delta K \\
\delta K & -\delta M
\end{array}\right)
$$

and write $\delta \underline{L}$ as

$$
\delta \underline{L}=\left(\begin{array}{lll}
\underline{0} & \underline{0} & \underline{0} \\
\underline{0} & \delta \underline{l} & \underline{0} \\
\underline{0} & \underline{0} & \underline{0}
\end{array}\right)
$$

In Eq. (33) $\delta \underline{l}$ occupies $(n, m)$ components of $\delta \underline{L}$, where $n$ and $m$ are either $N$ or $N+1$. For the coordination with Eq. (33) we also partition the matrix $\underline{G}$ and vector $\mathbf{U}$ in the same fashion,

$$
\underline{G}=\left(\begin{array}{lll}
\underline{G}_{11} & \underline{f} & \underline{G}_{13} \\
\underline{f}^{t} & \underline{g} & \underline{h}^{t} \\
\underline{G}_{13}^{t} & \underline{h} & \underline{G}_{33}
\end{array}\right), \quad \mathbf{U}=\left(\begin{array}{c}
\mathbf{v} \\
\mathbf{u} \\
\mathbf{w}
\end{array}\right),
$$

where $f, g$, and $\underline{h}$ are, respectively, $(N-1) \times 2,2 \times 2$, and $(N-1) \times 2$ matrices defined by

$$
\begin{aligned}
& \underline{f}=\left(\begin{array}{cc}
g_{N-1} & g_{N} \\
\vdots & \vdots \\
g_{1} & g_{2}
\end{array}\right), \\
& \underline{h}=\left(\begin{array}{cc}
g_{2} & g_{1} \\
\vdots & \vdots \\
g_{N} & g_{N-1}
\end{array}\right),
\end{aligned}
$$

and

$$
\mathbf{v}=\left(\begin{array}{c}
U_{-N+1} \\
\vdots \\
U_{-1}
\end{array}\right), \quad \mathbf{u}=\left(\begin{array}{c}
U_{0} \\
U_{1}
\end{array}\right), \quad \mathbf{w}=\left(\begin{array}{c}
U_{2} \\
\vdots \\
U_{N}
\end{array}\right)
$$

With the use of the quantities given by Eqs. (32)-(36), Eq. (31) reduces to

$$
\begin{aligned}
& (\underline{1}+\underline{g} \delta \underline{l}) \mathbf{u}=\mathbf{0}, \\
& \mathbf{v}=-\underline{f} \delta \underline{l} \cdot \mathbf{u}, \\
& \mathbf{w}=-\underline{h} \delta \underline{l} \cdot \mathbf{u},
\end{aligned}
$$

where 1 is now a $2 \times 2$ unit matrix. Thus the normalmode frequencies perturbed by the presence of the impurity cell and the vibrational amplitudes $\mathbf{u}$ at interfaces between the impurity cell and host cells are determined by solving Eq. (37). Once these quantities are obtained, the amplitudes $\mathbf{v}$ and $\mathbf{w}$ at other interfaces are calculated from Eqs. (38) and (39). We explicitly write Eq. (37) as

$$
\left(\begin{array}{cc}
1-g_{0} \delta J+g_{1} \delta K & -g_{1} \delta M+g_{0} \delta K \\
-g_{1} \delta J+g_{0} \delta K & 1-g_{0} \delta M+g_{1} \delta K
\end{array}\right)\left(\begin{array}{c}
U_{0} \\
U_{1}
\end{array}\right)=\left(\begin{array}{l}
0 \\
0
\end{array}\right)
$$

The solvability condition for this equation, i.e., $\operatorname{det}(\underline{1}+\underline{g} \delta \underline{l})=0$ leads after a bit of algebra to

$1+\delta K=0$

$g_{0} \Omega_{X}-g_{1}\left(\lambda_{A} \mu_{X}+\lambda_{X} \mu_{A}-\sigma_{A} \zeta_{X}-\sigma_{X} \zeta_{A}\right)=0$,

where we have used Eq. (23), and $\Omega_{X}(\omega)=\lambda_{X}+\mu_{X}$. As discussed by Maradudin et al. ${ }^{17}$ the solutions of Eq. (40) should give the frequencies of only those normal modes in a SL perturbed by the introduction of an impurity cell. We shall consider these two solutions in more detail.

According to Eq. (23), Eq. (41) means that $\sigma_{A}=0$ and hence $\lambda_{A} \mu_{A}=1$. This leads to $\left|\Omega_{A}(\omega)\right|>2$, that is, the perturbed frequency appears in the gaps of forbidden frequency of the host SL. However, the frequency satisfying $\sigma_{A}=0$ must be excluded as a solution of Eq. (19) from which Eq. (40) has been derived, because at this frequency at least one of $\sigma_{n}$ and $\sigma_{n-1}$ vanishes. Hence this solution should be considered more carefully. It is easily seen that if $n$ is a site of an $A$-type host cell $\operatorname{det} \underline{Y}_{n}=0$ holds at this frequency, and accordingly the inverse matrix $\underline{Y}_{n}^{-1}$ to $\underline{Y}_{n}$ used in Eq. (16) does not exist. Taking this result into account, we find that $U_{n}(n=0, \pm 1, \pm 2, \ldots)$ vanishes identically for $\sigma_{A}=0$. Thus the perturbation induced by the introduction of an impurity cell does not excite any vibration at the frequency predicted by Eq. (41) in a band gap of the host SL.

For an arbitrary choice of the impurity cell, Eq. (42) has to be solved only numerically. However, if we specialize to the following case of practical interest, a further insight into the characteristics of the impurity mode will be gained by analytic calculations. In view of the growing procedure of SL's the most easily obtainable impurity cell may consist of the same kind of constituents as the host cell, e.g., $x=a$ and $y=b$, but with different layer thickness. The impurity cell consisting of layers with different elastic properties from the host layers is generally possible. Unfortunately, impurity layers with large acoustic mismatch will also produce large lattice mismatch with the host layers, and therefore they are hard to grow properly. Accordingly, in what follows we assume that $x=a$ and $y=b$. Equation (42) is now reduced to 
$g_{0}\left[2 \cos \gamma \cos \delta-\left(\frac{Z_{b}}{Z_{a}}+\frac{Z_{a}}{Z_{b}}\right] \sin \gamma \sin \delta\right]=g_{1}\left[2 \cos (\alpha-\gamma) \cos (\beta-\delta)-\left(\frac{Z_{b}}{Z_{a}}+\frac{Z_{a}}{Z_{b}}\right] \sin (\alpha-\gamma) \sin (\beta-\delta)\right]$,

where $\gamma=k_{x} d_{x}=k_{a} d_{x}$ and $\delta=k_{y} d_{y}=k_{b} d_{y}$. This equation is still too complicated to be analyzed as it stands. The evaluation of Eq. (43), however, simplifies greatly in the following two cases.

(a) $d_{b}=d_{y}$ and $d_{a} \neq d_{x}$, i.e., $\beta=\delta$ and $\alpha \neq \gamma$. This means that one of the layers comprising the impurity cell is exactly the same as one of the constituent layers in the host SL. Thus this is equivalent to assuming a single layer as the impurity cell. Note that the structure of this impure SL is symmetric with respect to the impurity layer. Now, Eq. (43) is simplified as

$$
g_{0} \Omega_{X}=2 g_{1} \cos (\alpha-\gamma) \text {. }
$$

Substituting Eq. (30) for $g_{0}$ and $g_{1}$ into Eq. (44), we have

$$
\cos (\alpha-\gamma)=\frac{\Omega_{X}(\omega)}{\Omega_{A}(\omega) \mp\left|\Omega_{A}^{2}(\omega)-4\right|^{1 / 2}}, \quad\left|\Omega_{A}(\omega)\right|>2
$$

where the negative (positive) sign is applied for $\Omega_{A}>2$ $\left(\Omega_{A}<-2\right)$. Because the modulus of the denominator of Eq. (45) is smaller than 2 for $\omega$ in a frequency gap of the host SL, i.e., $\left|\Omega_{A}(\omega)\right|>2$, Eq. (45) has a solution only when $\left|\Omega_{X}(\omega)\right|<2$ is satisfied. This indicates that the perturbed frequency cannot exist in a region that is a common gap for both the pure $A$-type SL and the pure $X$ type SL. This is exactly what the Saxon-Hutner theorem states. ${ }^{21}$

(b) $d_{x}=d_{a} / 2$ and $d_{y}=d_{b} / 2$. In this particularly simple case the thickness of each layer in the impurity cell is equal to one-half of the corresponding layer thickness in the host cell, and hence $\gamma=\alpha-\gamma$ and $\delta=\beta-\delta$. Equation (43) now leads to

$$
\Omega_{X}(\omega)=0
$$

since $g_{0}=g_{1}$ does not hold. It is easily proven that for $\omega$ satisfying this condition, $\Omega_{A}(\omega)<-2$ and the perturbed frequency appears only in the zone-boundary gaps of the host SL. The absence of the impurity-mode frequency in the zone-center gaps is also the consequence of the Saxon-Hutner theorem. The conditions $\alpha=2 \gamma$ and $\beta=2 \delta$ imply that the width of the folded Brillouin zone in the pure $A$-type SL is a half of that in the pure $X$-type SL. Accordingly, the frequencies in the zone-center gaps in the host SL are always within the gaps of the pure $X$ type SL, and they lie in the spectral gaps of any disordered SL consisting of $\boldsymbol{A}$-type and $X$-type cells.

The displacement amplitudes at interfaces away from the impurity cell are determined by Eqs. (38) and (39) in terms of the impurity-mode frequency and amplitudes $U_{0}$ and $U_{1}$ given by Eq. (37). Explicitly, they are written for $n>2$ as
$U_{n}=g_{n}\left(\delta J U_{0}-\delta K U_{1}\right)+g_{n-1}\left(-\delta K U_{0}+\delta M U_{1}\right)$,

$U_{-n+1}=g_{n}\left(-\delta K U_{0}+\delta M U_{1}\right)+g_{n-1}\left(\delta J U_{0}-\delta K U_{1}\right)$

As we have already noted, for $\omega$ satisfying $\left|\Omega_{A}\right|>2$, Green's function $g_{n}$ depends on $n$ as $g_{n} \sim \epsilon^{|n|}$. Accordingly, the displacement amplitude of cell interfaces which vibrates with frequencies in forbidden gaps of the host SL decays exponentially with increasing distance from the impurity cell. This localized characteristic of the displacement amplitude is quite similar to that in a linear chain with a defect atom. Note that the rate of exponential decay of $g_{n}$, or $U_{ \pm n}$, is greater, the greater the distance of $\omega$ is from band edges satisfying $\left|\Omega_{A}\right|=2$.

\section{NUMERICAL RESULTS}

\section{A. Phonon transmission rate}

In a perfect, periodic SL with an infinite repetition of the unit cell, the phonons with wave number at the center and the boundary of the folded zone are Bragg reflected and cannot be transmitted through it. This is related to the occurrence of frequency gaps in the phonon dispersion relation at the center and edge of the mini-Brillouin zone. For an actual SL with a finite number of periods the exact periodicity is lost, but there still exist distinct dips in transmission for phonons in those frequency gaps. These dips in transmission have been observed by phonon spectroscopy ${ }^{3,7,10}$ and phonon imaging. ${ }^{9,16}$ Hence, with regard to the experimental observability of the localized impurity states in SL's, we shall calculate numerically the phonon transmission rate in SL's involving an impurity cell. In Sec. II we have shown that isolated frequencies should appear in the forbidden gaps of the spectrum of the host SL, where significant dips are predicted in phonon transmission. Therefore, in a perturbed SL, we can expect that the local enhancement in transmission should occur in the corresponding frequency ranges, which evidences the existence of the impurity states. If the magnitude of this enhancement is large enough we can observe in principle the presence of the impurity mode in a SL by acoustic-phonon transmission experiments with quasimonochromatic phonon detectors.

The numerical results for the frequency dependence of transmission rate are shown in Figs. 2 and 3 for longitudinal-acoustic (LA) phonons propagating in the growth direction of (111)-AlAs/GaAs SL's. ${ }^{22}$ The calculation of transmission rate has been published elsewhere. ${ }^{9}$ Figure 2(a) exhibits a result corresponding to case (a) given in Sec. II, where an $A$-type host cell consists of 15 monolayers (ML's) of AlAs ( $a$ layer) and 6 ML's of GaAs 
( $b$ layer), while an $X$-type impurity cell consists of $8 \mathrm{ML}$ 's of AlAs ( $x$ layer) and 6 ML's of GaAs ( $y$ layer). ${ }^{23}$ The impurity cell is assumed to be sandwiched in between 30 $A$-type cells. Comparing with Fig. 2(b), which shows the transmission rate in the perfect SL with the impurity cell replaced by the host cell, the sharp enhancement in the transmission can be seen in the frequency ranges exhibiting strong dips. The frequencies calculated from Eq. (44), i.e., $v_{1}=0.433 \mathrm{THz}$ and $v_{2}=0.901 \mathrm{THz}$, coincide exactly with the ones at which those local enhancements in transmission occur in Fig. 2(a).

It is also recognized in Fig. 2(a) that no enhancement is present at all in the small dip in transmission at about 1.35 THz. This is the result of the Saxon-Hutner theorem. We note that for the layer thickness assumed in this example the pure $A$-type and $X$-type SL's have common frequency ranges in which their band gaps overlap each other. This happenes in every third frequency gap of the pure $A$-type SL.

The example for the LA-phonon transmission rate in a
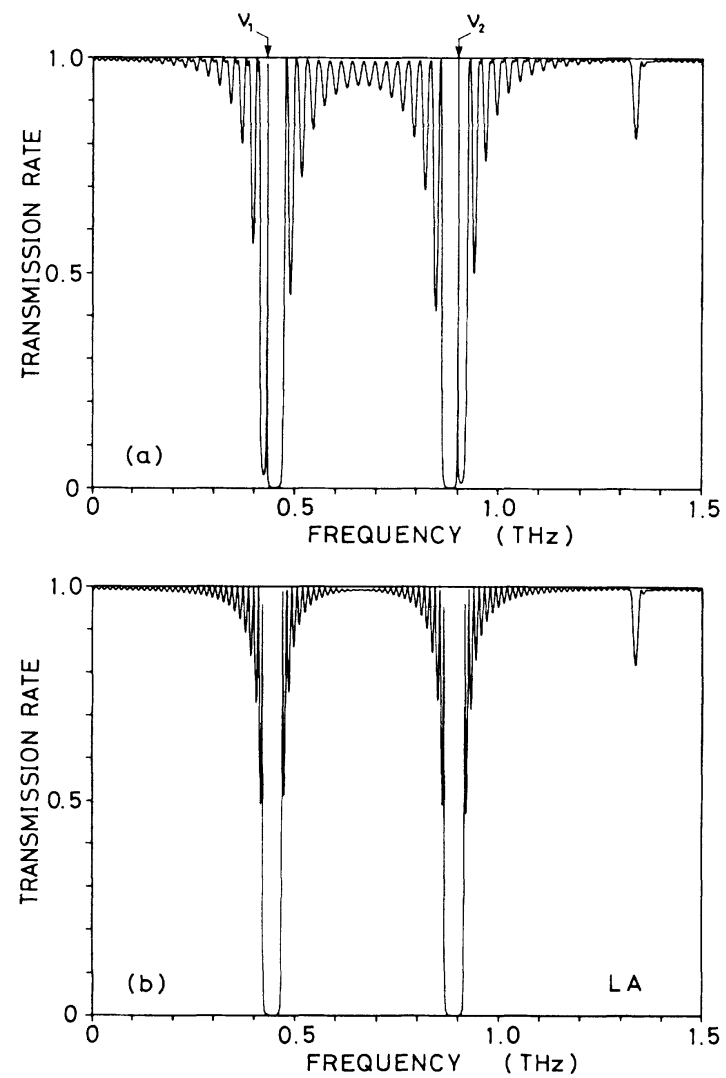

FIG. 2. Frequency dependence of LA-phonon transmission rate in (111)-AlAs/GaAs SL, (a) with an impurity cell and (b) without an impurity cell. The total number of cells is 31 . The host cell $(A)$ consists of 15 ML's of AlAs and 6 ML's of GaAs, and the impurity cell $(X)$ consists of 8 ML's of AlAs and 6 ML's of GaAs. In (a) the $X$ cell is located at the middle of $30 \mathrm{~A}$ cells. Frequencies $v_{1}=0.433 \mathrm{THz}$ and $v_{2}=0.901 \mathrm{THz}$ are calculated from Eq. (42) or (44).
(111)-AlAs/GaAs SL satisfying criterion (b) of Sec. II is given in Fig. 3(a). Here, we assume 12 and 14 ML's for the thickness of $a$ (AlAs) and $b$ (GaAs) layers of the $A$ type host cell, and 6 and 7 ML's are assumed for the thickness of $x$ (AlAs) and $y$ (GaAs) layers in the $X$-type impurity cell. The propagation configuration is the same as that in the previous case and $15 A$-type cells are assumed on both sides of the impurity cell. Comparing with Fig. 3(b), showing the transmission rate in the pure $A$-type SL, we also recognize the local enhancements in transmission at certain isolated frequencies in the first and third dips. We have again confirmed that these enhancements are really due to the impurity modes by checking the coincidence of the frequencies $v_{1}=0.342$ $\mathrm{THz}$ and $v_{2}=1.032 \mathrm{THz}$ calculated from Eq. (46) with those at which the enhancement in transmission occurs. It should be remarked that the absence of impurity-cell effects in the zone-center gaps, i.e., every second dip in transmission, observed in Fig. 3(a), is exactly in accord with the prediction made in Sec. II.
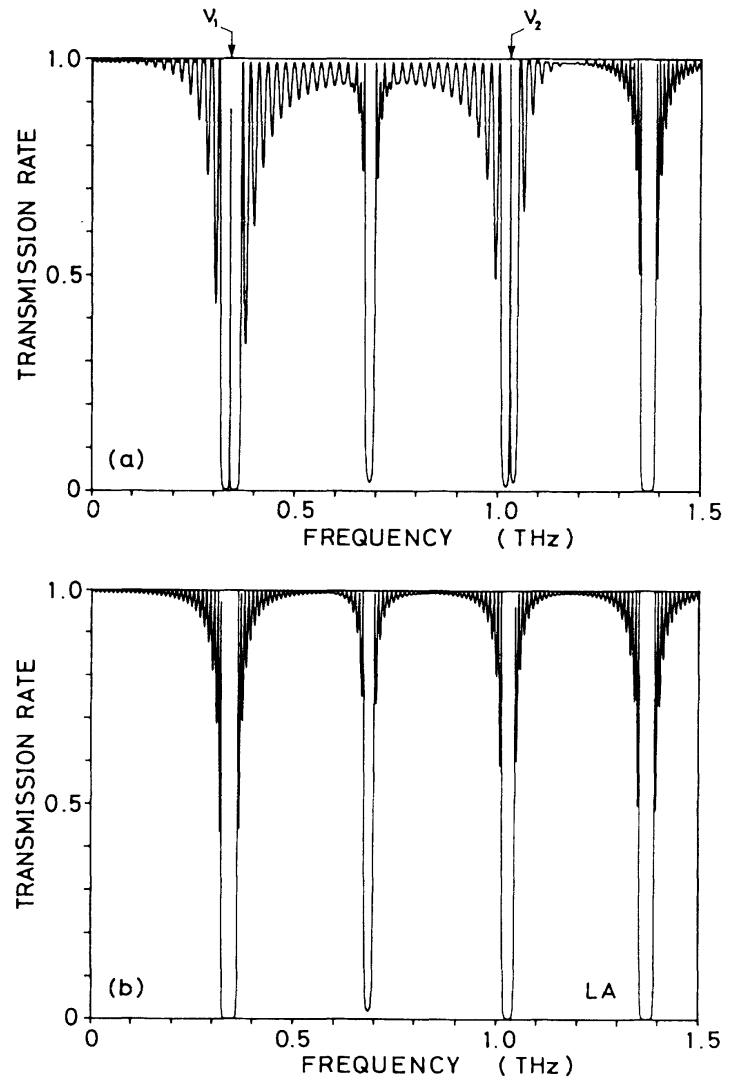

FIG. 3. Frequency dependence of LA-phonon transmission rate in (111)-AlAs/GaAs SL, (a) with an impurity cell and (b) without an impurity cell. The host cell $(A)$ consists of 12 ML's of AlAs and 14 ML's of GaAs, and the impurity cell $(X)$ consists of 6 ML's of AlAs and 7 ML's of GaAs. Cell configurations are the same as those in Fig. 2. Frequencies $v_{1}=0.342 \mathrm{THz}$ and $v_{2}=1.032 \mathrm{THz}$ are calculated from Eq. (42) or (46). 


\section{B. Amplitude profiles}

In Sec. II we have discussed that for frequencies satisfying $\left|\Omega_{A}\right|>2$ the Green's function $g_{n}$ decreases as $\epsilon^{|n|}$ $(|\epsilon|<1)$ with increasing $|n|$, i.e., the site number of interfaces counted away from the impurity cell. This result together with Eq. (47) leads to the localized nature of impurity-mode vibration in SL's analogous to the case of ordinary lattices. In order to see the localization characteristics we have plotted in Figs. 4 and 5 the profiles of the displacement amplitudes $U_{n}$ (as well as the amplitudes at interfaces inside the unit cells) at perturbed frequencies in SL's for which the local enhancement in transmission shown in Figs. 2 and 3 is obtained. The mode of the vibration is LA. Explicitly, those frequencies are again $v_{1}=0.433 \mathrm{THz}$ and $v_{2}=0.901 \mathrm{THz}$ for Figs. 4(a) and 4(b), and $v_{1}=0.324 \mathrm{THz}$ and $v_{2}=1.032$ THz for Figs. 5(a) and 5(b). For all these frequencies the localization of vibrational amplitudes near the defect cell is evident. The decay rates of these profiles depend on the distance of the corresponding frequency from the band edges. Here we note that the spatial structure of the SL for which Fig. 4 is obtained is symmetric with respect to the one of the impurity layers, and hence the amplitude profiles should be symmetric or antisymmetric with respect to the center of this layer. However, no such symmetry exists in the SL for which Fig. 5 is calcu-
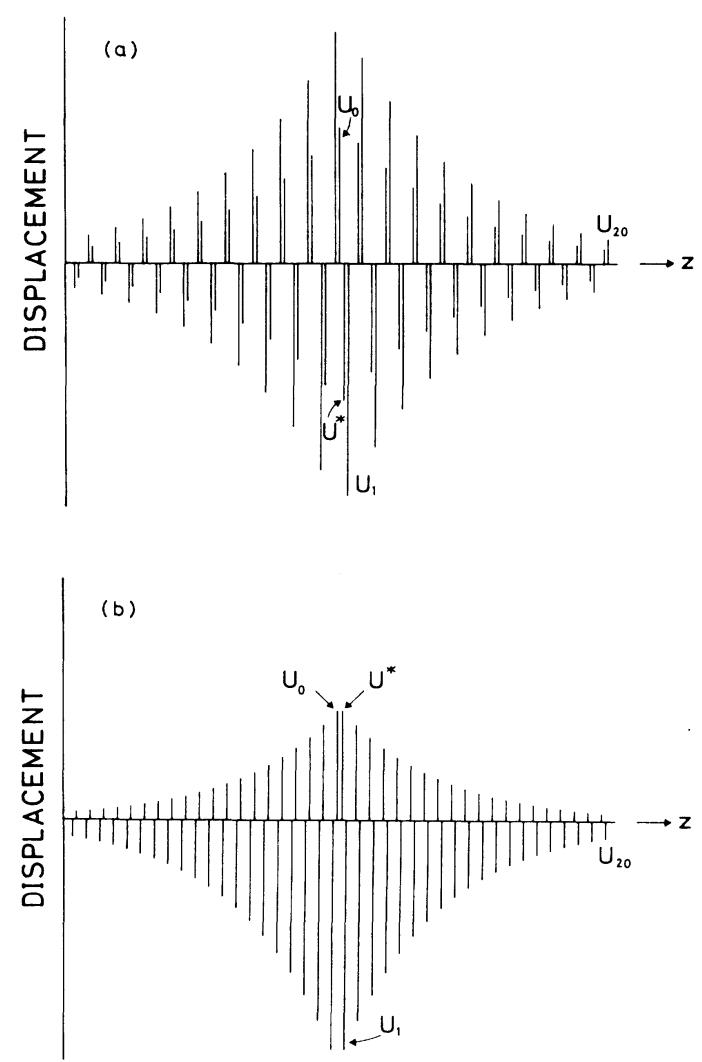

FIG. 4. Profiles of vibrational amplitudes at interfaces in the SL corresponding to Fig. 2(a). Frequencies are (a) $v_{1}=0.433$ $\mathrm{THz}$ and $(\mathrm{b}) v_{2}=0.901 \mathrm{THz}$. lated, and as a result the amplitude profiles in this figure do not reveal any symmetry as in Fig. 4.

\section{Transmission rate at oblique propagation}

So far, we have considered the effects of an impurity cell on the SL vibration with wave vector perpendicular to the interfaces of layers. For the vibration with wave vector oblique to the interfaces the situation becomes much more complicated, and it is generally difficult to predict the effects by analytic calculations. This is mainly because both the displacement and stress fields have three independent components, and the transfer matrix corresponding to Eq. (2) becomes a $6 \times 6$ instead of a $2 \times 2$ matrix. Even in this case, however, we may still expect the existence of localized vibrational states in the frequency gaps of the host SL.

In order to check this expectation we have numerically calculated the angular dependence of phonon transmission rate in a SL with an impurity cell. Here, we note that for a fixed frequency the transmission rate in a periodic SL exhibits certain characteristic features also in its angular dependence. The condition for Bragg reflection of phonons in a SL depends on their propagation angle as well as frequency. Hence the dips in transmission should occur at several angles satisfying the Bragg condition. The most interesting feature in the ob-
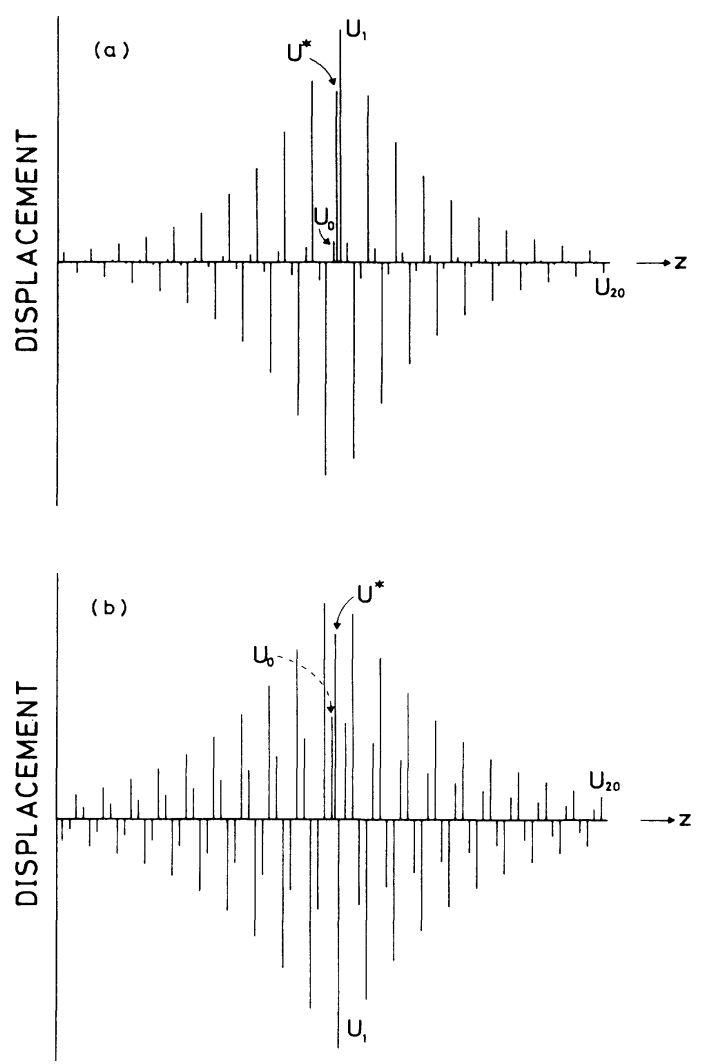

FIG. 5. Profiles of vibrational amplitudes at interfaces in the SL corresponding to Fig. 3(a). Frequencies are (a) $v_{1}=0.342$ $\mathrm{THz}$ and (b) $v_{2}=1.032 \mathrm{THz}$. 
lique phonon propagation in SL's is the possibility of intermode Bragg reflection in addition to ordinary intramode Bragg reflection, which yields a frequency gap of the spectrum inside the folded zone. ${ }^{8}$ This reflection of phonons causes extra dips in transmission. Thus, by scanning the propagation direction of phonons with a fixed frequency, one or more dips in transmission are generally obtained.

Figure 6(a) shows the calculated angular dependence of the transmission rate of $0.85-\mathrm{THz}$ LA phonons in a (001)-AlAs/GaAs SL. The thickness of both layers in the $A$-type host cell is assumed to be equal, i.e., 7 ML's. For the $X$-type impurity cell 7 and 14 ML's are assumed for the thicknesses of AlAs $(x)$ and GaAs $(y)$ layers, respectively. In Fig. 6 we denote by $\theta$ the polar angle in the plane rotated $22.5^{\circ}$ away from the (100) and (110) planes in the real space of GaAs. The $X$ cell is again assumed to be sandwiched in between $30 A$-type cells. For comparison's sake we have also plotted in Fig. 6(b) the corresponding transmission rate in the pure $A$-type SL with 31 cells. The relatively narrow dip in Fig. 6(b) at about $\tan \theta=0.8$ is due to the first-order intramode Bragg reflection yielding the zone-boundary gap in the dispersion relation of the LA mode. The broader dip in
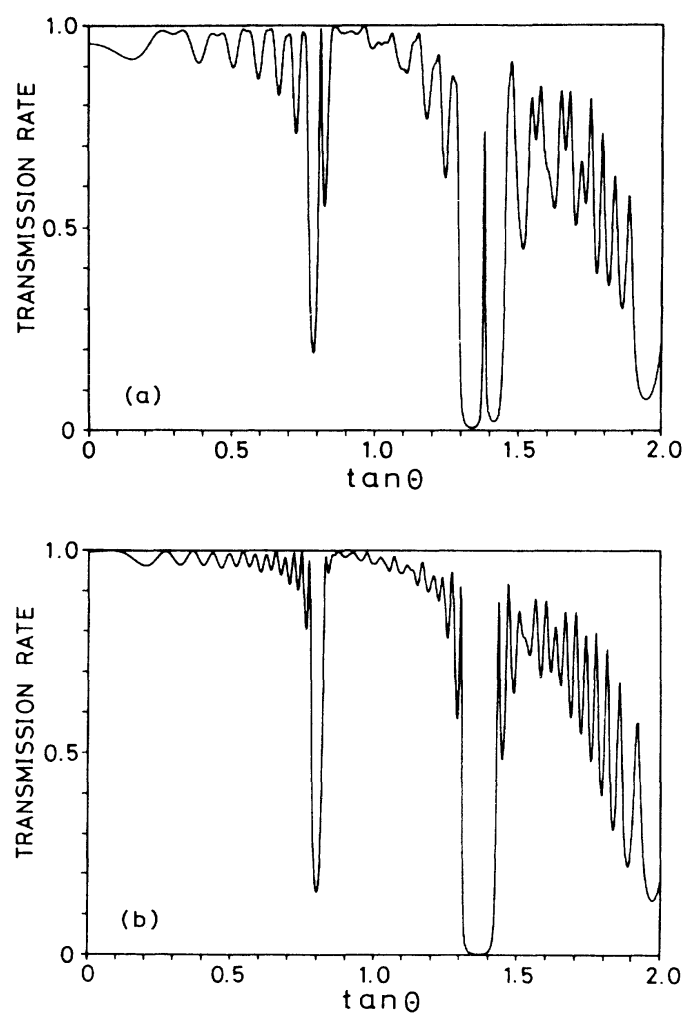

FIG. 6. Angular dependence of LA-phonon transmission in (001)-AlAs/GaAs SL, (a) with an impurity cell and (b) without an impurity cell. The total number of cells is 31 . The host cell $(A)$ consists of 7 ML's of AlAs and 7 ML's of GaAs, and the impurity cell $(X)$ consists of 7 ML's of AlAs and 14 ML's of GaAs. In (a) the $X$ cell is located at the middle of $30 \mathrm{~A}$ cells. transmission found at about $\tan \theta=1.4$ is due to the intermode Bragg reflection of LA phonons into fast transverse phonons. Comparing Figs. 6(a) and 6(b), we find that the local enhancement in transmission similar to the ones in Figs. 2(a) and 3(a) exists in both dips in transmission. This result suggests that the localized vibrational mode occurs also within the intrazone frequency gaps of the host SL due to intermode Bragg reflection.

\section{CONCLUSIONS}

An appropriately light-impurity atom added substitutionally to a lattice is responsible for a spatially localized vibration at a frequency above or in a gap in the host vibrational spectrum. In this paper we have examined the presence of the same kind of localized vibrations in an impure SL by deriving and exploiting a difference equation governing the motion of cell interfaces. In the several cases of practical interest we have found that the localized states appear in the frequency gaps of the host $\mathrm{SL}$ as far as the condition for the Saxon-Hutner theorem is not satisfied. Those frequency gaps are not restricted to the ones at the center and edge of the folded zone originating from the intramode phonon-Bragg reflection. The existence of the localized states in the intrazone frequency gaps due to intermode phonon-Bragg reflection is also suggested but only numerically by the presence of local enhancement in transmission. The detection of the enhancement in transmission predicted at frequencies in the spectral gaps of the host SL should provide an experimental verification of the localized modes in SL's. A quasimonochromatic phonon detector is needed to resolve the existence of the enhanced transmission in relatively narrow frequency ranges.

For frequencies in the allowed bands of a host SL, the impurity cell causes the scattering, or reflection of phonons which are otherwise transmitted perfectly. In this case $g_{n}$ is complex, and there is a possibility of the resonance scattering of phonons. ${ }^{17}$ It should occur at frequencies $\omega_{r}$ for which the real part of Eq. (42) vanishes. Whether or not the actual resonance occurs depends on whether or not the width of the assumed resonance is much smaller than $\omega_{r}$. For the combination of layers to be allowed by the growth condition of the SL, the possibility for the true resonance to occur would be small. In fact, we cannot see any evidence of the resonance scattering in Figs. 2 and 3. The detailed study on this problem is currently under way.

To conclude, the localized vibrational modes generally exist in frequency gaps of the host SL irrespective of the propagation direction and modes participating in the Bragg reflection responsible for these spectral gaps.

\section{ACKNOWLEDGMENTS}

The author would like to thank T. Sakuma for helpful discussions. He is also indebted to $\mathrm{N}$. Nishiguchi for useful comments on the manuscript. This work is partially supported by a Grant-in-Aid for Scientific Research from the Ministry of Education, Science and Culture, Japan. 
${ }^{1}$ J. L. Merz, A: S. Barker, Jr., and A. C. Gossard, Appl. Phys. Lett. 31, 111 (1977).

${ }^{2}$ A. S. Barker, Jr., J. L. Merz, and A. C. Gossard, Phys. Rev. B 17, 3181 (1978).

${ }^{3}$ V. Narayanamurti, H. L. Störmer, M. A. Chin, A. C. Gossard, and W. Wiegmann, Phys. Rev. Lett. 43, 2012 (1979).

${ }^{4}$ C. Colvard, R. Merlin, M. V. Klein, and A. C. Gossard, Phys. Rev. Lett. 45, 298 (1980); C. Colvard, T. A. Gant, M. V. Klein, R. Merlin, R. Fischer, H. Morkoc, and A. C. Gossard, Phys. Rev. B 31, 2080 (1985).

${ }^{5}$ R. E. Camley, B. Djafari-Rouhani, L. Dobryzynski, and A. A. Maradudin, Phys. Rev. B 27, 7318 (1983).

${ }^{6}$ S. K. Yip and Y. C. Chang, Phys. Rev. B 30, 7037 (1984).

${ }^{7}$ O. Koblinger, J. Mebert, E. Dittrich, S. Döttinger, W. Eisenmenger, P. V. Santos, and L. Ley, Phys. Rev. B 35, 9372 (1987).

${ }^{8}$ S. Tamura and J. P. Wolfe, Phys. Rev. B 35, 2528 (1987).

${ }^{9}$ D. C. Hurley, S. Tamura, J. P. Wolfe, and H. Morkoc, Phys. Rev. Lett. 58, 2446 (1987); S. Tamura, D. C. Hurley, and J. P. Wolfe, Phys. Rev. B 38, 1427 (1988); S. Tamura and J. P. Wolfe, ibid. 38, 5610 (1988).

${ }^{10}$ P. V. Santos, J. Mebert, O. Koblinger, and L. Ley, Phys. Rev. B 36, 1306 (1987).

${ }^{11}$ S. Ren, H. Chu, and Y. C. Chang, Phys. Rev. Lett. 59, 1841 (1987); Phys. Rev. B 37, 8899 (1988).

${ }^{12}$ H. Chu, S. Ren, and Y. C. Chang, Phys. Rev. B 37, 10746
(1988).

${ }^{13}$ R. Merlin, K. Bajema, R. Clarke, F.-Y. Juang, and P. K. Bhattacharya, Phys. Rev. Lett. 55, 1768 (1985).

${ }^{14}$ M. W. C. Dharma-wardana, A. H. MacDonald, D. J. Lockwood, J.-M. Barbibeau, and D. C. Houghton. Phys. Rev. Lett. 58, 1761 (1987).

${ }^{15}$ S. Tamura and J. P. Wolfe, Phys. Rev. B 36, 3491 (1987).

${ }^{16}$ D. C. Hurley, S. Tamura, J. P. Wolfe, K. Ploog, and J. Nagle, Phys. Rev. B 37, 8829 (1988).

${ }^{17}$ See, for example, A. A. Maradudin, E. W. Montroll, G. H. Weiss, and I. P. Ipatova, Theory of Lattice Dynamics in the Harmonic Approximation (Academic, New York, 1971), Chap. 8, and references therein.

${ }^{18}$ S. M. Rytov, Zh. Eksp. Teor. Fiz. 29, 605 (1955) [Sov. Phys. - JETP 2, 466 (1956)].

${ }^{19}$ M. Kohmoto, Phys. Rev. B 34, 5043 (1986).

${ }^{20}$ J. S. Langer, J. Math. Phys. 2, 584 (1961).

${ }^{21}$ See, for example, J. M. Ziman, Models of Disorder (Cambridge University Press, London, 1979).

${ }^{22}$ The energy flux of LA phonons is focused in the [111] direction in both GaAs and AlAs.

${ }^{23}$ The thickness of one monolayer is $3.26 \AA$ in the [111] direction and $2.83 \AA$ in the [100] direction for both GaAs and AlAs. Mass density and longitudinal sound velocity in the [111] direction are $5.36 \mathrm{~g} / \mathrm{cm}^{3}$ and $5.37 \mathrm{~km} / \mathrm{s}$ for GaAs, and $3.76 \mathrm{~g} / \mathrm{cm}^{3}$ and $6.45 \mathrm{~km} / \mathrm{s}$ for AlAs. 